\title{
Trapping Electrons in a Room-Temperature Microwave Paul Trap
}

\author{
Clemens Matthiesen $\odot$, Qian Yu®, Jinen Guo®, Alberto M. Alonso, and Hartmut Häffner ${ }^{*}$ \\ Department of Physics, University of California, Berkeley, California 94720, USA
}

(Received 13 May 2020; revised 11 December 2020; accepted 17 December 2020; published 29 January 2021)

\begin{abstract}
We demonstrate trapping of electrons in a millimeter-sized quadrupole Paul trap driven at $1.6 \mathrm{GHz}$ in a room-temperature ultrahigh vacuum setup. Cold electrons are introduced into the trap by ionization of atomic calcium via Rydberg states and stay confined by microwave and static electric fields for several tens of milliseconds. A fraction of these electrons remain trapped longer and show no measurable loss for measurement times up to a second. Electronic excitation of the motion reveals secular frequencies that can be tuned over a range of several tens to hundreds of MHz. Operating a similar electron Paul trap in a cryogenic environment may provide a platform for all-electric quantum computing with trapped electron spin qubits.
\end{abstract}

DOI: 10.1103/PhysRevX.11.011019

\section{INTRODUCTION}

The spin-up and spin-down states of an electron form the archetypal two-level system in quantum physics and make the electron a natural candidate for realizing a quantum bit. Quantum computing approaches use electrons in both condensed matter and atomic systems-for instance, confined in quantum dots or bound to donors in semiconductors [1-3], or bound as valence electrons in trapped atomic ions $[4,5]$. In these examples, the confinement to either the host solid-state environment or to a much heavier ion can limit the potential of the electron spin qubit: for trapped ions, entanglement is typically mediated by the slow motion of the heavy ions in a shared trapping potential $[6,7]$, which limits the gate speed, while in condensed matter systems, unwanted coupling of the electron's charge and magnetic moment to the imperfect environment limits coherence times.

An approach that promises to remove these limitations is to confine individual free electrons in actual vacuum [8-10]. Here, we show experimentally that this can be achieved with the type of traps used for the currently most-advanced ion-trap quantum computers, namely, quadrupole Paul traps. Compared to commonly trapped ions, the electron's charge-to-mass ratio is larger by a factor $10^{4}-10^{5}$, such that motion-based gates and shuttling operations could be sped up by 2 orders of magnitude.

\footnotetext{
*hhaeffner@berkeley.edu
}

Published by the American Physical Society under the terms of the Creative Commons Attribution 4.0 International license. Further distribution of this work must maintain attribution to the author(s) and the published article's title, journal citation, and DOI.
Subject Areas: Atomic and Molecular Physics,

Quantum Information
Based on measurements for ion ground-state qubits, which should experience similar decoherence mechanisms to trapped electron spin qubits, coherence times of at least one second are expected [11]. Furthermore, reducing the complex level structure down to a minimum of two levels rules out qubit errors due to population leakage [12]. Adapting the quantum-CCD architecture developed for trapped ions [13] to trapped electrons offers the opportunity to build a fast, modular, and high-fidelity quantum computer using advanced microwave technology [14-17], which promises better compatibility with current microfabrication methods compared to laser technology and optical beam delivery.

Beyond quantum computing, the experimental platform we introduce here may offer new avenues for creating and studying small cold plasma [18], highly controllable fewto single-electron sources for electron optics applications [19], or single-electron mechanical oscillators [20].

Trapping single electrons in vacuum has previously been achieved in two other platforms. First, electrons were confined in cryogenic Penning traps in the early 1970s [21] by combining a large magnetic field and a constant electric quadrupole field. While several proposals have considered using single electrons in Penning traps as qubits [22-24], limited work has been performed on experimental realizations so far [25]. Electrons can also be trapped above the surface of liquid helium, offering quantum information applications in milli-Kelvin environments [26,27], and recent experimental efforts have reached the single-electron regime [28].

Our approach to trapping electrons builds on the established quadrupole radio-frequency ion-trap architecture, which is at the forefront of current quantum computing approaches with atomic ions [4,5]. Guiding electrons along 
a radio-frequency guide [29] has been achieved, and electrons have been cotrapped with ions in a combined Paul and Penning trap [30], but trapping electrons in a pure Paul trap has not been reported so far. While potential applications to quantum computing will require cryogenic environments [8-10], we concentrate here on demonstrating electron trapping in a proof-of-principle experiment at room temperature.

Paul traps employ a rapidly oscillating quadrupole electric field to confine charged particles at the null of the quadrupole field in two or three dimensions. The effective confining potential can be described by the pseudopotential $U_{\mathrm{p}}=\left(q^{2} E^{2} / 4 m \Omega^{2}\right)$, where $E$ and $\Omega$ are the amplitude and frequency of the oscillating electric field, and $q$ and $m$ the charge and mass of the trapped particle(s). The spatial dependence $U_{\mathrm{p}}(x, y, z)$ derives from the quadrupole electric field amplitude $E(x, y, z)$. The stability of trajectories for a charged particle in a quadrupole trap can be described with the $a_{\mathrm{M}}$ and $q_{\mathrm{M}}$ parameters of the Mathieu equation, which are known as the stability parameters in the context of ion traps and have been studied theoretically and experimentally [31,32]. Typically, trajectories in the trap are stable if the frequency of motion $\omega$ of the particles in the potential is much slower than the frequency $\Omega$ of the confining field and if the pseudopotential depth, defined as the maximum of the pseudopotential, is much larger than the kinetic energy of the charged particles. While the Mathieu equation provides useful intuition for quadrupole traps, it should be noted that the pseudopotential picture and the treatment with the Mathieu equation are no longer accurate when the potential deviates from a purely harmonic dependence.

There are three main challenges to moving from ion to electron trapping. First, because of the lower electron mass, the trapping field must be at higher frequencies. The stability parameters $\left(a_{\mathrm{M}}\right.$ and $\left.q_{\mathrm{M}}\right)$ and depth of a quadrupole trap scale as $\left(m \Omega^{2}\right)^{-1}$, requiring the drive frequency $\Omega$ for an electron trap to be about 2 orders of magnitude higher than for typical ion traps. Second, electrons must be created with energies low enough to stay confined by the trapping potential. We require that cold electrons are injected directly into the trap center and that the trap is sufficiently deep. Third, in the absence of fluorescence detection, we need a different mechanism to evidence trapping.

\section{EXPERIMENT DESIGN}

\section{A. Electron trap}

We begin by describing the microwave quadrupole trap engineered for this experiment, shown in an exploded view in Fig. 1. It consists of three double-sided printed circuit boards (PCBs) separated by alumina spacers. The central board features a coplanar $\lambda / 2$ waveguide resonator capacitively coupled to a microwave feedline (right-hand side of the PCB). The end of the resonator in the board center

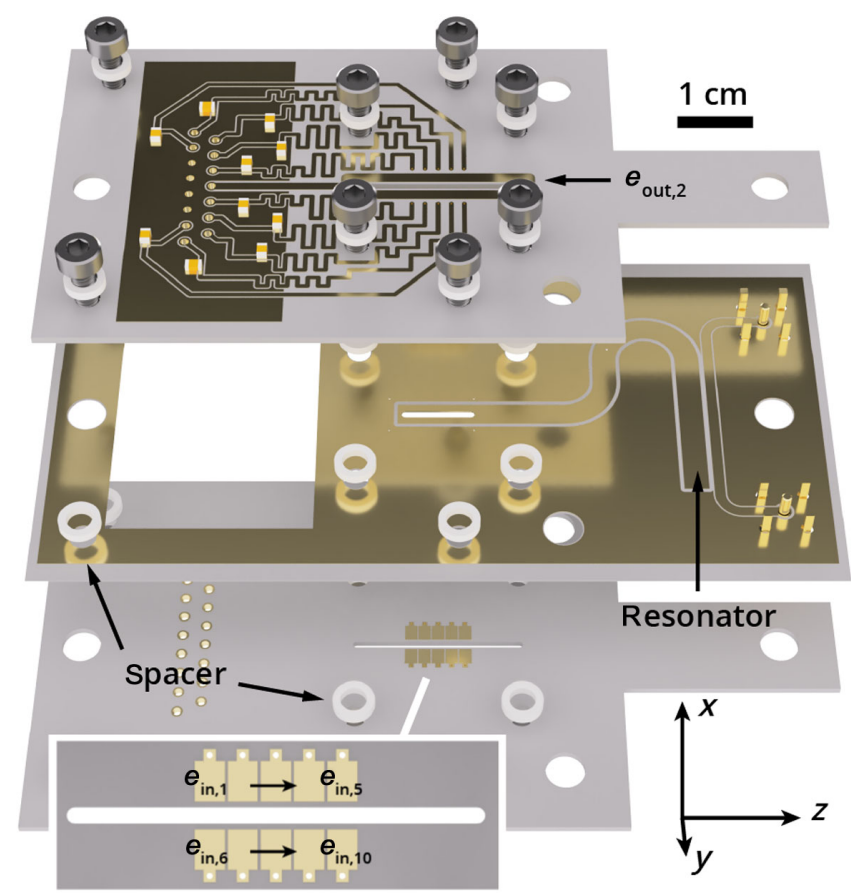

FIG. 1. Electron trap design. Exploded view of trap printed circuit boards. Driving the half-wave coplanar waveguide resonator (central board) gives rise to a quadrupole microwave trap inside the slot at the central end of the resonator. The two outside boards are identical and feature electrodes $e_{\text {in,1-10 }}\left(e_{\text {in,11-20 }}\right)$ on the bottom (top) to provide static confinement along the slot direction (see bottom inset for electrode labels). The boards are separated with alumina spacers of $1.27 \mathrm{~mm}$ height and have a footprint of about 5-by-10 $\mathrm{cm}$.

contains a slot and functions as the trap's microwave electrode, providing an ac quadrupole field that confines electrons inside the slot in the $x$ and $y$ directions. The quality factor of the resonator is about 35 . When fully assembled and connected inside the ultrahigh vacuum (UHV) chamber, we measure a resonance frequency of $2 \pi \times 1.60 \mathrm{GHz}$ and find we can reach about $100 \mathrm{~V}$ on the microwave resonator with $5-\mathrm{W}$ input power. Integrating a coplanar resonator into the trap design provides a convenient solution to reaching the high frequencies needed for electron trapping, and future cryogenic experiments can take advantage of previous work on waveguide resonators in the context of superconducting qubits [33,34]. The resonator is held at de ground potential via a tap in its center, which connects it to the grounded top surface of the board. The outside PCBs, mirroring each other about the central board, each feature ten rectangular electrodes along the slot on the inside board surface, labeled $e_{\mathrm{in}, j}$, with $j=1-20$. Electrodes $e_{\mathrm{in}, 1-10}$ are visible on the lower board and magnified in the inset, while electrodes $e_{\mathrm{in}, 11-20}$ are on the hidden side of the upper board. The traces delivering voltages to the electrodes are on the outside surfaces, visible for the top board, and linked to a ground electrode via $10-\mathrm{pF}$ decoupling capacitors. Both boards also feature a 

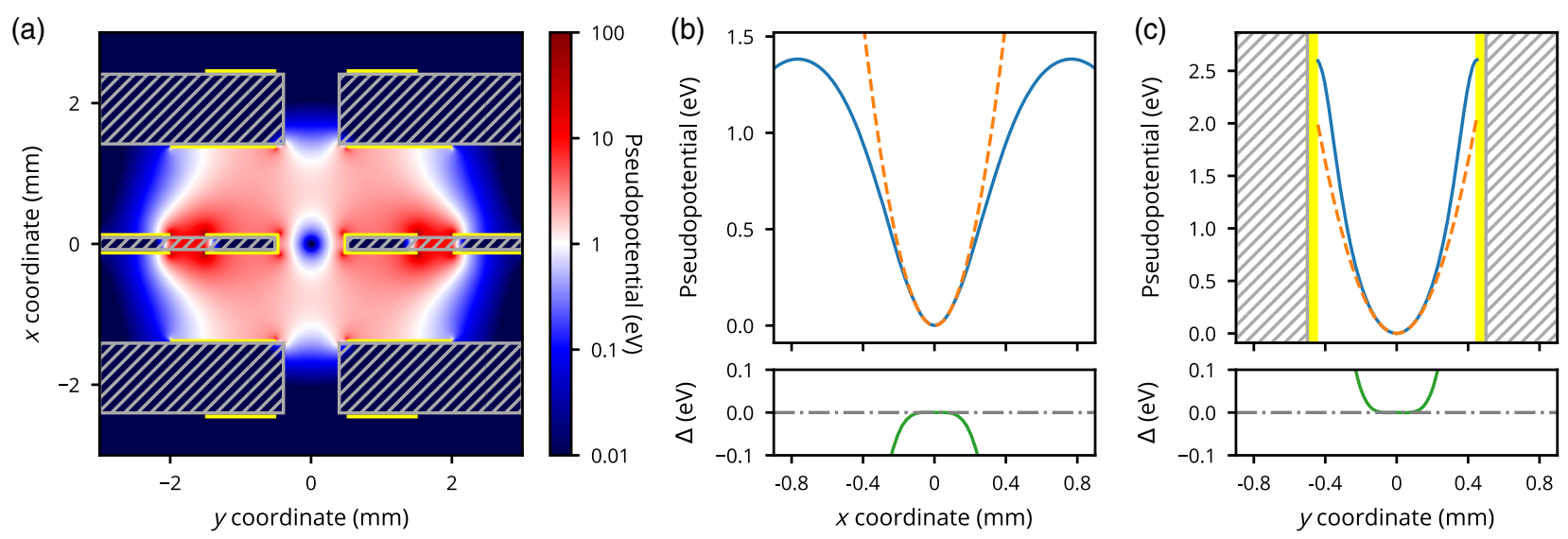

FIG. 2. Electron trapping potential. (a) Contour plot of the trapping potential based on the pseudopotential approximation in the $x y$ plane in the center of the slot for $90-\mathrm{V}$ amplitude at 1.6-GHz frequency on the resonator electrode. The circuit board substrate is indicated by the hashed areas; metal electrodes are highlighted in yellow. (b) Top: pseudopotential along the $x$ axis through the trap center (blue continuous curve), compared to an ideal harmonic potential (dashed orange). Bottom: deviation $\Delta$ of the pseudopotential from the harmonic potential. (c) Top: pseudopotential along the $y$ axis through the trap center (blue continuous curve), compared to an ideal harmonic potential (dashed orange). Location of the trap substrate (electrodes) shown as the hashed grey (solid yellow) area. Bottom: deviation $\Delta$ of the pseudopotential from the harmonic potential.

single electrode that surrounds the slot on the outside surface and is labeled $e_{\mathrm{out}, 1}$ for the bottom board and $e_{\mathrm{out}, 2}$ for the top board. Electrodes $e_{\text {in, } 1-20}$ are used to apply a static quadrupole field, confining electrons in the $z$ (axial) direction, while $e_{\text {out,1-2 }}$ are held at the dc ground potential. Wires soldered to the outer boards supply dc voltages in the \pm 28 -V range from a 16-bit digital-to-analog converter, while the microwave voltage is applied via SMA connectors to the central board.

Figure 2 details the trap pseudopotential experienced by an electron when the resonator supplies $90 \mathrm{~V}$ at $2 \pi \times$ 1.6 GHz and all dc electrodes are grounded. Figure 2(a) displays a map of the pseudopotential for a cut through the trap in the $x y$ plane at the center of the slot, highlighting the trap substrate and electrodes as hashed grey and yellow areas, respectively. In Figs. 2(b) and 2(c), we show that the trap depth based on the pseudopotential approximation (continuous blue curves) is about $1.3 \mathrm{eV}$ (or $15000 \mathrm{~K}$ ), limited by the weaker confinement along the $x$ direction. We compare the pseudopotential to an ideal harmonic potential (orange dashed curve) and find they match closely to a distance of about $100 \mu \mathrm{m}$ from the trap center, as exemplified by the green curves in the bottom panels, which show their difference $\Delta$. The secular frequency for an electron moving in this potential (radial modes of motion) corresponds to about $2 \pi \times 300 \mathrm{MHz}$.

\section{B. Electron loading and detection}

With a suitable trap design in place, we address the challenges of injecting electrons into the trap and detecting them. Previous experiments involving trapping electrons in Penning traps or guiding electrons in a linear quadrupole potential employed electron guns, either as a primary electron source [29] or to create secondary electrons through collision ionization of background gas [30,35]. Here, we borrow the two-stage procedure for photoionization (PI) of calcium, which is used for trapping ions from an atomic beam [36]. It enables both the creation of very cold electrons by tuning the lasers close to the ionization threshold and preferential ionization in the trapping region by optical alignment. Since fewer charged particles are introduced around the trap using this method, we also reduce accidental charging of the trap, which would modify the trapping potential. Detection is accomplished by applying voltage pulses to several dc electrodes, which distort the trapping potential to extract trapped electrons and accelerate them into a microchannel plate detector (MCP).

\section{Experiment setup and protocol}

The main components of the experimental setup and their alignment in the UHV chamber are shown in Fig. 3(a), omitting electric leads for simplicity. The base pressure in the chamber is below $1 \times 10^{-10}$ mbar. In addition to the trap itself, the chamber contains a resistive oven aligned to direct an atomic calcium beam through the trap slots when heated (steel tube labeled "oven"), the two-stage MCP, and a steel mesh, which directs electrons extracted from the trap towards the MCP. The PI laser beams (423- and 390-nm wavelength) are overlapped and traverse the chamber at right angles to the $\mathrm{Ca}$ oven, focusing near the trapping region with a beam waist of about $30 \mu \mathrm{m}$. The $423-\mathrm{nm}$ single-mode laser is tuned to be on resonance with the neutral calcium $4^{1} S_{0}-4^{1} P_{1}$ transition, while the freerunning multimode 390 -nm laser diode is tuned by temperature and current to maximize the electron ionization rate. While the $4^{1} P_{1}$-continuum ionization threshold is at about 


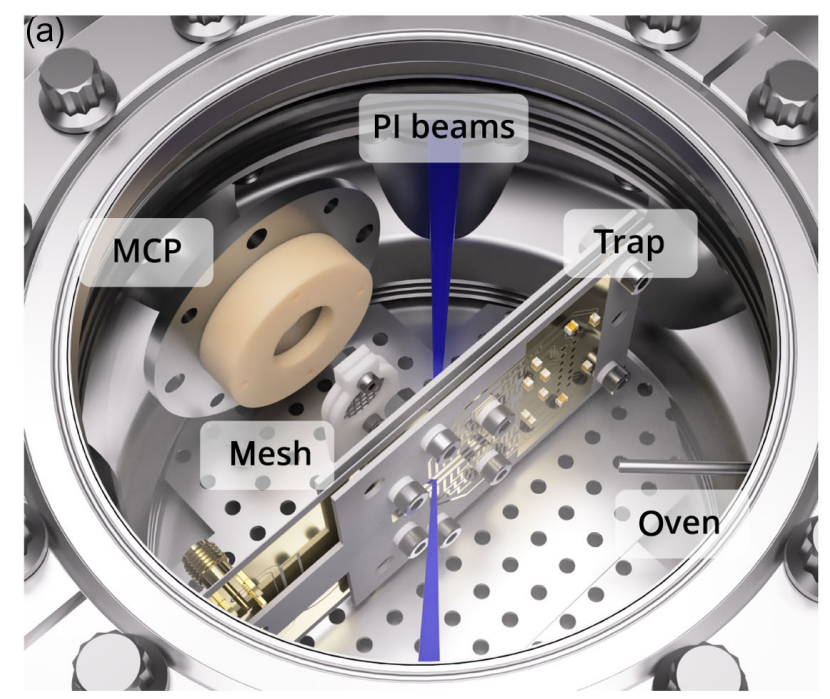

(b)

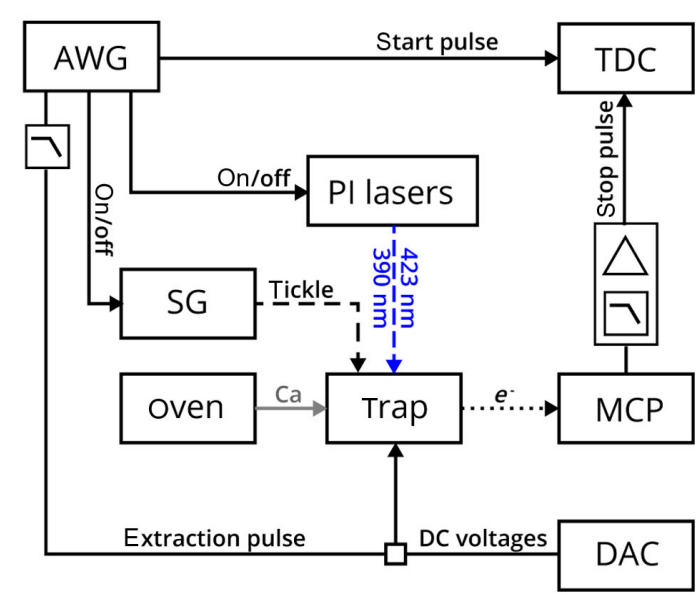

FIG. 3. Experimental setup and control schematic. (a) Simplified rendering of the setup inside the UHV chamber, showing the electron trap, a microchannel plate (MCP) detector, a mesh, the path of the PI beams, and an oven supplying calcium for ionization. (b) Key elements for experiment synchronization. An arbitrary waveform generator (AWG) provides the extraction pulses to the trap and the start signal for a time-to-digital converter (TDC). It controls the timing for pulsing on and off the PI loading beams and a signal generator (SG), which excites the electron motion ("tickle"). Pulses from the MCP provide the stop signals to the TDC. Low-pass filters prevent electronic pickup of the microwave trap drive and the extraction pulses by the MCP detection circuit, which contains pulse shaping and amplification elements. The Ca oven and a digital-to-analog converter (DAC) for the dc trap voltages are operated with constant settings during an experiment.

$389.8 \mathrm{~nm}$, we find the ionization rate peaks when the diode center wavelength is about $390.3 \pm 0.2 \mathrm{~nm}$, suggesting ionization is taking place via Rydberg states [36]. As such, we expect electrons to inherit only minimal kinetic energy from the ionization process, and rather, their energy in the trapping potential is determined by their ionization location and the phase of the microwave field. We note that since the PI lasers are copropagating, $\mathrm{Ca}$ ionization conditions are met over an extended volume, which follows the laser beam path, and the majority of electrons are created outside of the trap. Constraints on optical access to the trap in our particular setup prevent us from aligning the two PI beams such that they only intersect inside the trap.

Figure 3(b) shows a schematic of the electronics setup for synchronizing the experiment. An AWG functions as the experiment clock, providing the trap extraction pulse and the start signal triggering a TDC. The extraction pulses are added to the static voltages for dc confinement that originate from a DAC. The AWG further controls the timing for switching on and off both the 390-nm PI laser for loading and a SG used to apply a rf tone (labeled "tickle") to the trap. The electron detection signal is picked off from the MCP anode supply voltage with a high-pass filter, shaped and amplified so that it can be used as the TDC stop signal. Low-pass filtering the extraction pulses $(2 \pi \times 50$ $\mathrm{MHz}$ cutoff) prevents electronic pickup at the MCP, and a further low-pass filter $(2 \pi \times 200-\mathrm{MHz}$ cutoff $)$ in front of the shaping and amplification circuit removes pickup at the frequency of the microwave drive.
The timing for one experimental cycle is illustrated in Fig. 4(a). It starts with a loading phase of variable duration $t_{\text {load }}$, where the 390-nm PI laser is pulsed on. The 423-nm PI laser is kept on during the full cycle. Loading is followed by a variable time $t_{\text {wait }}$, where we either keep all settings constant or apply a rf tone at frequency $\omega_{\text {tickle }}$ to electrode $e_{\text {in, 17 }}$. Finally, an extraction pulse of 20-ns duration is applied to three electrodes, $e_{\text {out }, 1}$ with $14 \mathrm{~V}$ amplitude, and $e_{\text {in, } 3,8}$ with $10 \mathrm{~V}$ amplitude, which eject trapped charges from the trap. For the experiments presented here, we supply a constant current to the calcium oven and keep the microwave trap drive in continuous-wave mode such that the voltage amplitude on the microwave resonator corresponds to about $90 \mathrm{~V}$. We use about $500 \mu \mathrm{W}$ of $423-\mathrm{nm}$ laser light and approximately $2.4 \mathrm{~mW}$ of $390-\mathrm{nm}$ light for the photoionization process. The mesh is at $150-\mathrm{V}$ potential, while the first and second stages and the anode of the MCP are kept at $200 \mathrm{~V}, 2200 \mathrm{~V}$, and $2500 \mathrm{~V}$, respectively.

In Fig. 4(b), we show a histogram of MCP detection events, where the loading and wait times are $t_{\text {load }}=t_{\text {wait }} \approx$ $10 \mu \mathrm{s}$. Data are displayed as the probability to record an event during a 1-ns time bin, and we acquire data for $10^{7}$ experimental cycles. During the loading period, we observe a small constant signal mirroring the shape of the 390-nm laser pulse, likely from electrons that were just created but not trapped. Application of the extraction pulse at the end of the experimental cycle results in a large and sharply localized signal from the MCP, demonstrating that electrons remain in the trap $10 \mu \mathrm{s}$ after the end of the loading pulse. 


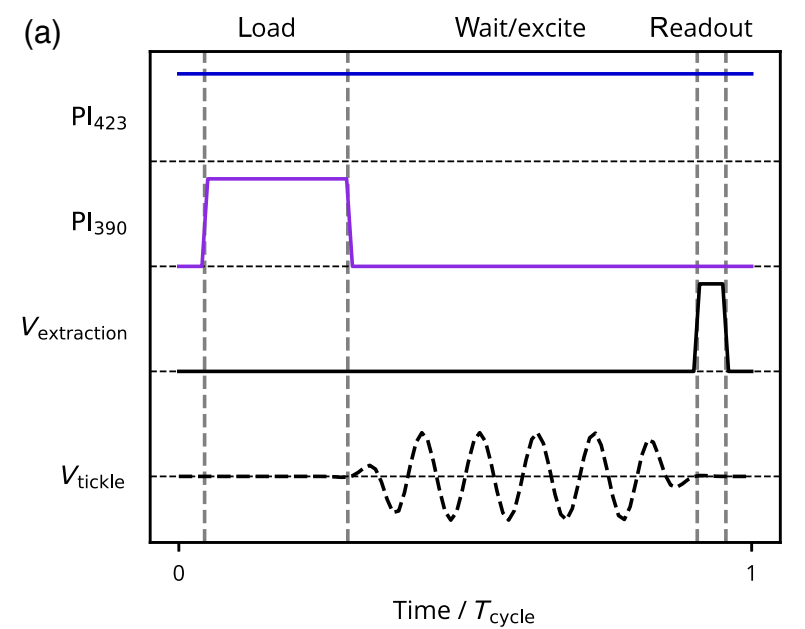

(b)

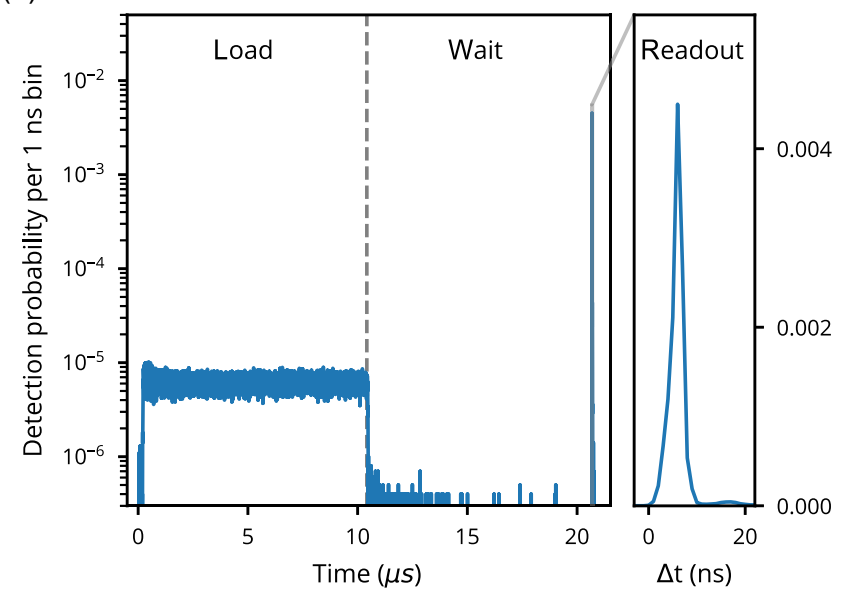

FIG. 4. Measurement protocol and typical data. (a) Illustration of one cycle of the experimental protocol. Free electrons are created during the loading phase when the 390-nm laser is switched on. A rf tone is applied to one dc electrode in some experiments during the "wait/excite" phase. Voltage pulses to three dc electrodes in the "readout" phase eject electrons in the direction of the mesh and MCP. (b) Histogram of MCP detection events for experiment with load and wait times $t_{\text {load }}=t_{\text {wait }} \approx$ $10 \mu$ s. During loading, some untrapped electrons are accelerated into the MCP, replicating the 390-nm laser pulse shape. The extraction pulse empties the trap into the MCP, resulting in a large and sharply localized signal. Inset: close-up of the histogram during the readout phase.

The inset displays a close-up of the readout signal, which peaks with a full width at half maximum of about $2 \mathrm{~ns}$. Note that the inset uses a linear scale for the readout signal, while the full cycle is displayed using a semilogarithmic scale to show the background during loading as well.

\section{RESULTS}

\section{A. Electron loading and storage}

Having demonstrated electron trapping, we next quantify the trapping process. To investigate electron loading, we use the protocol introduced in Fig. 4(a) and vary $t_{\text {load }}$ for a fixed wait time $t_{\text {wait }}=10 \mu \mathrm{s}$. For ease of presentation, we sum detections over a 50-ns-wide window around the readout signal; see Fig. 5(a) for the results. The left ordinate displays the fraction of cycles with at least one detection pulse from the MCP, which approaches unity with a time constant $\tau_{1 / e} \approx 80 \mu \mathrm{s}$ as $t_{\text {load }}$ increases. We employ a simple threshold method to detect MCP pulses with the TDC and set a 60-ns deadtime following each detection to prevent double counting some events due to voltage ringing, which sets a natural limit of one detection event per cycle. Considering electron loss at the mesh and the MCP, we estimate that about one in eight extracted electrons results in a signal from the MCP. Taking into account the loss during the readout process and the fraction of detections per cycle, we can estimate the average number of electrons in the trap for each measurement setting (see the Appendix A for details). The right ordinate in Fig. 5(a) shows that the electron number is proportional to the loading time: It takes, on average, $10 \mu \mathrm{s}$ to load one electron, and we trap about 20 electrons for a loading time of $200 \mu \mathrm{s}$.

To measure the electron storage time in the trap, we set the loading time such that the trap rarely contains more than a single electron, and we record the readout signal as a function of the wait time; see Fig. 5(b) for the data. The measurement shows two distinct regimes, where about three-quarters of electrons are lost within $100 \mathrm{~ms}$ (exponential decay with $\tau_{1 / e}=30 \pm 7 \mathrm{~ms}$ for this measurement), while the remaining one-quarter of electrons show no detectable loss after $1 \mathrm{~s}$. The dark horizontal dash-dotted line displays the background detection level measured independently to be about $1 \times 10^{-4}$ detections per cycle.

Long storage times in the trap are essential if trapped electrons are to be used as qubits, so understanding the mechanism behind the loss is an important task. The dominant loss mechanisms for laser-cooled ions are collisions with background gas. Collisions - in particular, with heavier atoms and molecules - can provide sufficient energy to kick an ion out of its trapping potential or may lead to the formation of molecules. Given the light mass of the electron, one might expect collisional loss and electron capture to play a major role in our trap, too. In order to quantify this loss channel, we change the pressure in the vacuum chamber by more than an order of magnitude and measure the decay constant at chamber pressures of about $5 \times 10^{-10}$ mbar and $2 \times 10^{-8} \mathrm{mbar}$. We find no change within our measurement uncertainty (see Appendix $C$ for details), which rules out collision with the background gas as the primary loss channel.

The important difference between electrons in our trap and laser-cooled ions is their light mass, as well as the absence of a cooling mechanism, which dampens the motion and concentrates particles in the trap center. 

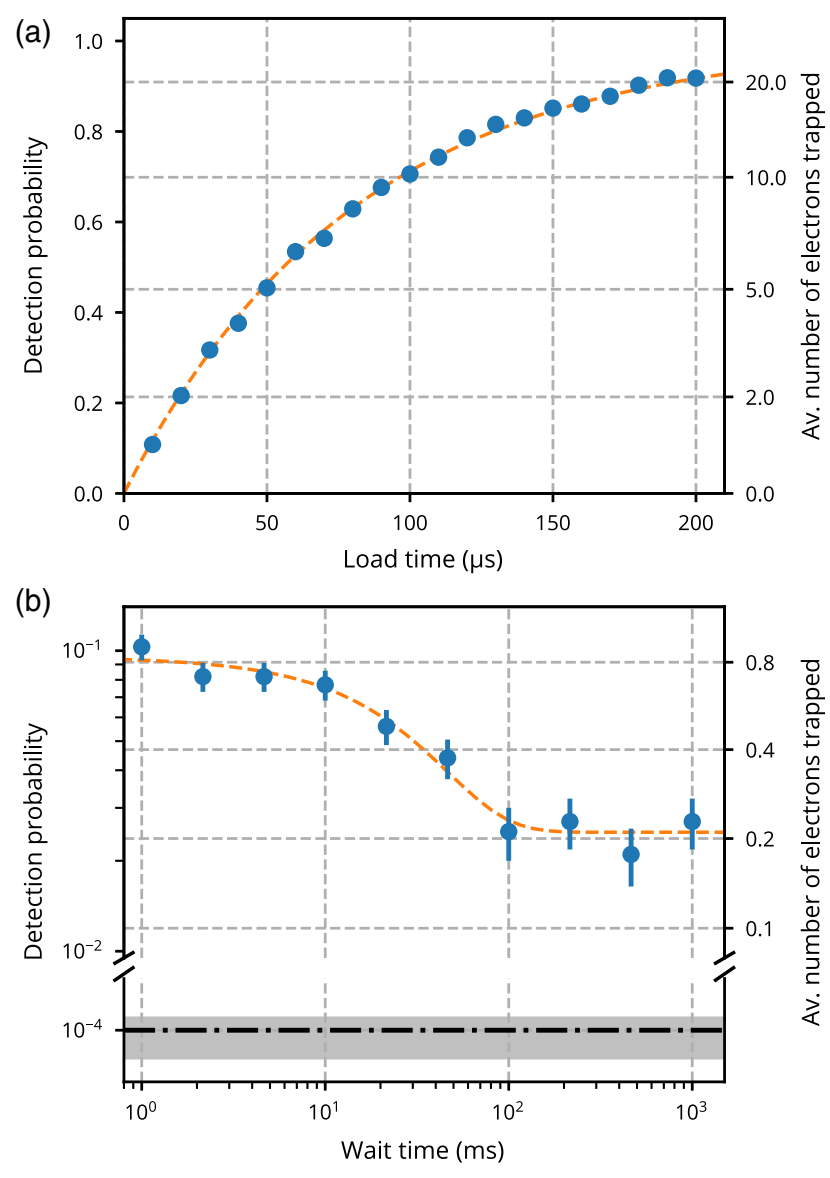

FIG. 5. Trapped electron loading and storage. (a) Electron trapping and detection probability as a function of loading time with $t_{\text {wait }}=10 \mu \mathrm{s}$. The dashed curve shows an exponential fit to $1-\exp \left(t / \tau_{1 / e}\right)$, with $\tau_{1 / e}=80.3 \pm 0.5 \mu \mathrm{s}$. Error bars due to statistical uncertainty are too small to be visible. (b) Storage time measurement with a double-exponential fit, showing threequarters of electrons leave the trap with a decay constant $\tau_{1 / e}=30 \pm 7 \mathrm{~ms}$, while the remaining quarter show no decay within measurement uncertainty. The horizontal dash-dotted line displays the background detection level based on an independent measurement. Error bars and the grey band correspond to 1 s.d. statistical uncertainty.

Electrons in our trap sample a much greater volume and hence experience anharmonicities in the trapping potential further away from the trap center. While the general motion of a charged particle in an anharmonic ac potential is nontrivial [37], we can make two general distinctions from the harmonic case. First, the normal modes of motion become coupled, and second, nonlinear resonances [38,39] enable the transfer of energy from the driven micromotion to the secular motion, leading to heating and particle loss. We illustrate particle loss for the motion of a single electron along one dimension of the trap in numerical simulations in Appendix $\mathrm{C} 2$ and find that trajectories for electrons ionized further than about $100 \mu \mathrm{m}$ from the trap center are generally not stable. The electron motion amplitude in the trap exceeds $250 \mu \mathrm{m}$ for those cases. This length scale is consistent with the onset of strong deviations of the pseudopotential from a purely harmonic form, as shown in Fig. 2. Within $200 \mu \mathrm{m}$ of the trap center, the pseudopotential deviates by less than $2 \%$ from the ideal harmonic potential, and one may expect stable motion there. Coupling between the secular modes and repeated perturbations can still heat electrons from stable into unstable orbits and lead to loss, however, since there is no damping of the motion. Such perturbations to the trapping potentials happen in our experiments, for instance, due to power fluctuations of the microwave field, charging of the trap substrate, or the interactions of multiple charges in the trap.

We can experimentally probe some elements of this argument: By changing the focusing and alignment of the PI beams, which affect the average ionization distance from the trap center, we find that larger PI beams in the trapping region are correlated with fewer long-lived electrons. We also observe that storage times decrease slightly when we increase the electron density in the trap by increasing the loading time.

We believe that trap anharmonicity is the driving force behind electron loss in our trap. Only the trajectories for single electrons confined within the harmonic trapping region are stable, so with the ionization volume dictated by the alignment of the photoionization beams, we expect that the majority of initially trapped electrons (which are created outside of the central $100 \mu \mathrm{m}$ ) will experience a sufficiently anharmonic potential to be driven out of the trap eventually. The tail of long-lived electrons in Fig. 5(b) is then attributed to the single electrons that remain in a stable trajectory after all other particles have been heated out of the trap.

Studying the loss mechanisms of trapped electrons in greater detail with this Paul trap will likely be an important subject for future work. However, the long lifetimes observed here show that heating effects-for instance, due to collisions with background gas or the electron micromotion [40,41] — are not prohibitive to conducting experiments even at room temperature. This result is an encouraging sign, in particular, for the prospects of nondestructive electron detection and cooling via imagecurrent measurements in a cryogenic environment. Studying trap loss may also yield insights relevant for quadrupole ion traps, and since the electron motion is faster by a factor of 100 compared to ion motion in a typical trap, experiments would take less time.

\section{B. Trap frequencies}

Finally, we are interested in the frequencies of the electron motion in the trap. Again, we follow the experimental protocol from Fig. 4(a), now loading about ten electrons on average and setting the wait time to $t_{\text {wait }}=2 \mathrm{~ms}$. During the wait time, we try to excite the motion of trapped electrons with a rf tone at frequency $\omega_{\text {tickle }}$. We step $\omega_{\text {tickle }}$ in increments of $1 \mathrm{MHz}$ from 20 to 

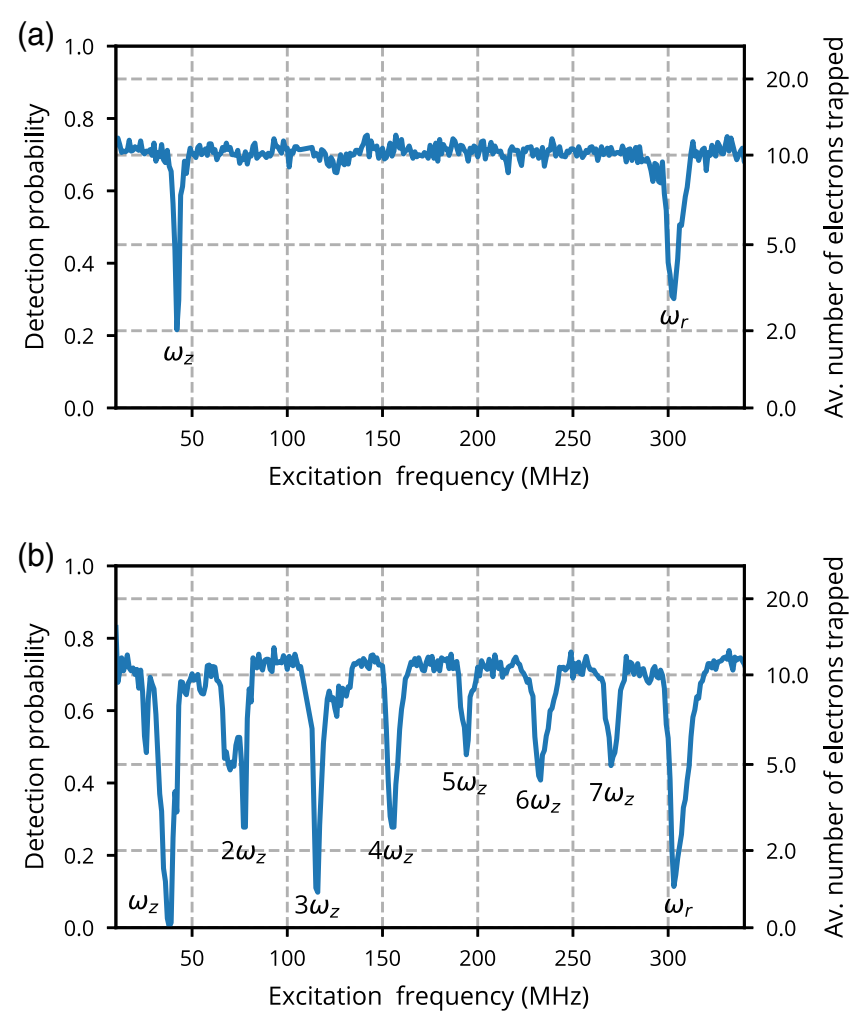

FIG. 6. Trap frequencies. Measurements of the motional resonances for an average of ten electrons loaded into the trap, and a wait time $t_{\text {wait }}=2 \mathrm{~ms}$ during which a rf tickle is switched on. The axial (radial) resonance is denoted $\omega_{z}\left(\omega_{r}\right)$. (a) The $5-\mathrm{mV}$ tickle excitation; (b) 20-mV tickle excitation.

$350 \mathrm{MHz}$ and monitor electron loss, which is indicative of a motional resonance. The top panel in Fig. 6 shows the electron loss spectrum for a tickle voltage of about $5 \mathrm{mV}$ applied to $e_{\mathrm{in}, 17}$, and it features two prominent dips. We can identify the resonances based on their response to dc and microwave voltages, revealing the dip at about $2 \pi \times$ $40 \mathrm{MHz}$ as the axial mode, while the $2 \pi \times 300-\mathrm{MHz}$ resonance is due to one of the radial modes of motion. We find no evidence of multielectron Wigner crystal modes, which is consistent with having weakly or noninteracting electrons with a range of energies in the trap.

For low-excitation tickle powers, only the fundamental resonances are visible. Exciting the system more strongly reveals a series of harmonics of the axial mode [see Fig. 6(b)] and a small shift in the fundamental frequency. Changing the dc and microwave voltages, we can tune the axial mode frequency between 30 and $100 \mathrm{MHz}$ and the radial mode between 200 and $380 \mathrm{MHz}$, limited by the voltage sources used in the experiment.

\section{CONCLUSIONS AND OUTLOOK}

In summary, we have presented the first experiment to trap electrons in a microwave Paul trap. Electrons can be loaded in tens of microseconds, and $25 \%$ survive up to at least one second. Trap frequencies ranging from several $10 \mathrm{MHz}$ to several $100 \mathrm{MHz}$ have been measured.

Trapping electrons in a Paul trap opens the door to using their unique properties for quantum information processing. One of the main challenges moving towards this goal is to cool the secular modes sufficiently to be able to perform quantum operations on them. The well-established method of detecting the electron image current with a resonant circuit provides a convenient cooling mechanism, as it thermalizes the detected mode of motion with the temperature of the detection circuit [21]. Some form of cryogenic cooling is then necessary to reach low electron temperatures. This approach may not appear straightforward considering the high voltages at microwave frequencies required for trapping, but several features may be used to our advantage. First, we do not, in principle, require the trap itself to be cold, just the detection circuit, which can be separate from the main body of the trap. Since the imagecurrent detection dissipates much less power than the microwave electrodes, cooling the detection circuit separately may be a sensible choice. Second, we do not need to reach the motional ground state to be in the quantum regime [42]. For the sake of concreteness, reducing the dimensions of the current trap by a factor of 10 , such that the trap center would be about $50 \mu \mathrm{m}$ from the nearest electrode, and increasing the drive frequency by a factor of $10(\Omega \sim 16 \mathrm{GHz})$, we would obtain radial modes at $\omega \sim 3 \mathrm{GHz}$. With a detection circuit cooled to $1.5 \mathrm{~K}$ and tuned to a radial mode, we expect an average mode occupation of 10 quanta, which is comparable to the mode occupation of a Doppler-cooled ion. In Ref. [9], we discussed schemes to cool other modes of motion and perform state readout under similar conditions.

Along these lines, the next milestones towards quantum control of trapped electrons would be nondestructive electron detection [21] and spin readout [9], which benefit from motional frequencies in the $\mathrm{GHz}$ regime (that is, smaller traps) and integration into a cryogenic environment. Building on technology that has already been demonstrated for quantum control of trapped ion hyperfine [14-17] and Zeeman [11] qubits could accelerate the development of a trapped electron quantum computing platform. Distribution of entanglement over large distances is another challenge for future studies. Here, dipole-dipole coupling of single electrons, or electron crystals, in separate traps is an attractive option to realizing entanglement over intermediate distances $[43,44]$. A path towards coupling electron qubits over longer distances could be via image currents in shared electrodes [45,46]. Techniques like these may enable the creation of large entangled states.

We also note that an electron in the harmonic potential of a Paul trap realizes an instance of the lightest possible electromechanical oscillator [47]. The resonance frequency and quality factor can be engineered by controlling the confining potential. While we believe a platform operating 
with trapped electrons as the sole qubit modality is the least challenging route, the ability to fine-tune the frequency of motion in situ and the electron's strong interaction with electric fields could be used for coupling to other quantum systems with resonances in the $\mathrm{GHz}$ range, such as superconducting qubits $[10,48]$. Finally, electron Paul traps may also find applications outside the realm of quantum information science. Our trap could, for instance, trap positrons and be employed for the preparation of antihydrogen $[49,50]$. Other applications include electric-field sensing at $\mathrm{GHz}$ frequencies [51], and using cold trapped electrons for imaging [19] or for plasma physics studies [18].

\section{ACKNOWLEDGMENTS}

We would like to thank S. Mouradian for feedback on the manuscript, the Yao Lab at University of California, Berkeley for loan of the TDC, and Dr. David E. Root, Keysight Laboratories, for initiating financial support and technical assistance by Keysight Technologies through the Keysight University Research Collaborations Program. A. M. A. was sponsored by the U.S. Department of Energy, Office of Science, Office of Basic Energy Sciences under Award No. DE-SC0019376.

\section{APPENDIX A: MCP DETECTION EFFICIENCY}

To be registered in our readout scheme, electrons have to escape the trap, pass the mesh, impinge on the MCP, and trigger the electron multiplication process. From electron trajectory simulations, we estimate the extraction process to be close to lossless; hence, the open area of the mesh and the nonunity MCP efficiency are the main loss factors. The open area of the mesh is 0.5 . The MCP detection efficiency depends on the energy of incoming electrons, which is determined by the potential difference between the trap and the first MCP stage, and the voltages we apply to the second stage and the anode of the MCP. For sufficiently high MCP voltages and electron energies of a few hundred $\mathrm{eV}$, the efficiency should saturate approximately at the MCP open area of about 0.6 , such that the upper bound on electron detection efficiency would be about $1 / 3$. We performed electron loading measurements like the one shown in Fig. 5(a) with a range of MCP voltages to calibrate the $\mathrm{MCP}$ detection efficiency for the operating conditions used in the main body of the paper.

Figure 7 shows the data (symbols) and fits to $1-$ $\exp \left(t / \tau_{1 / e}\right) \quad$ (dashed curves). The legend displays the MCP voltages and the fit parameter $\tau_{1 / e}$. We note that the $\tau_{1 / e}$ values are different from the data in Fig. 5(a) as the intensity of the photoionization beams was lower here. We observe a clear saturation of the detection efficiency as the voltages approach $500 \mathrm{~V}, 2500 \mathrm{~V}$, and $2800 \mathrm{~V}$ for the first stage, second state, and anode of the MCP, respectively. For these voltages, the MCP efficiency should correspond to

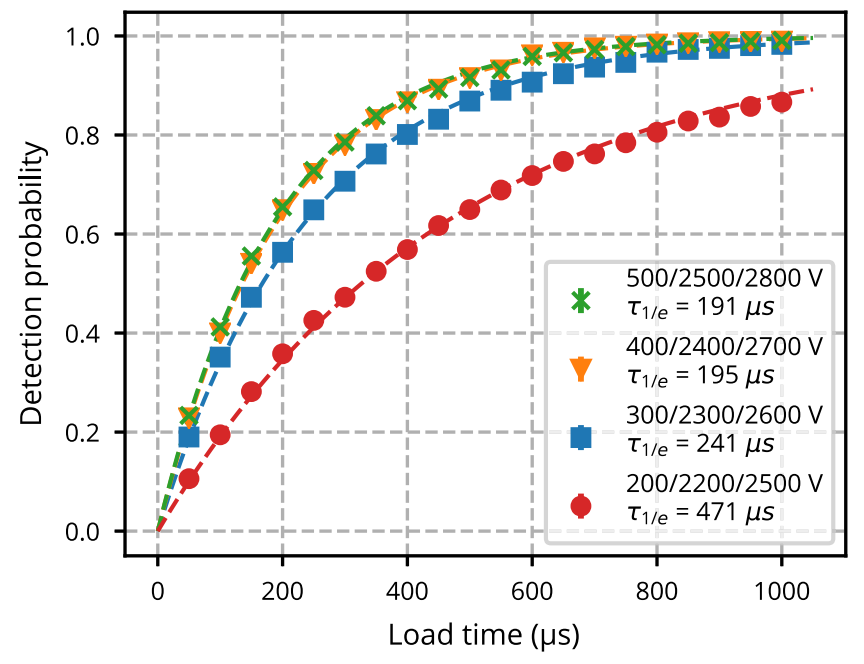

FIG. 7. Calibration of relative MCP detection efficiency. Measurement of electron loading rates [compare to Fig. 5(a)] for a range of $\mathrm{MCP}$ voltages. For typical operating conditions in this work $(200 / 2200 / 2500 \mathrm{~V})$, the detection efficiency is about $40 \%$ of the maximum efficiency.

the open area of 0.6, and it follows that for the typical operating conditions used here, the efficiency is reduced by a factor of about 2.5. Overall, we then estimate that $12 \%$ of electrons leaving the trap in the extraction process generate a detectable signal from the MCP.

A caveat in this estimate is that we are not accounting for possible degradation of the MCP, which would lower the efficiency somewhat.

\section{APPENDIX B: ELECTRON NUMBER ESTIMATE}

The conversion of measured detection probability to electron number assumes that electron detection events at the MCP follow Poissonian statistics. Concretely, we find the mean $\lambda$ for a Poisson distribution $P_{\mathrm{P}}(X, \lambda)$ such that $P_{\mathrm{P}}(0, \lambda)$ matches the measured probability to record no events during readout $P($ !detection $)=1-P$ (detection) . The product of $\lambda$ and the electron loss (inverse of detection efficiency) provides an estimate of the electron number in the trap at the point when the extraction sequence is applied.

\section{APPENDIX C: SUPPLEMENTAL DETAILS ON ELECTRON LOSS}

\section{Influence of collisions}

An ion pump with a line of sight to the main vacuum chamber is used to keep the pressure below the $1 \times 10^{-10} \mathrm{mbar}$ level. We found that operating the MCP while the ion pump is switched on leads to background detections in excess of 10 kilocounts per second. When the Ca oven is hot and the trap microwave drive is on, the pressure in the chamber increases to about $5 \times 10^{-10} \mathrm{mbar}$, and the electron detections due to the ion pump increase by 1-2 orders of 


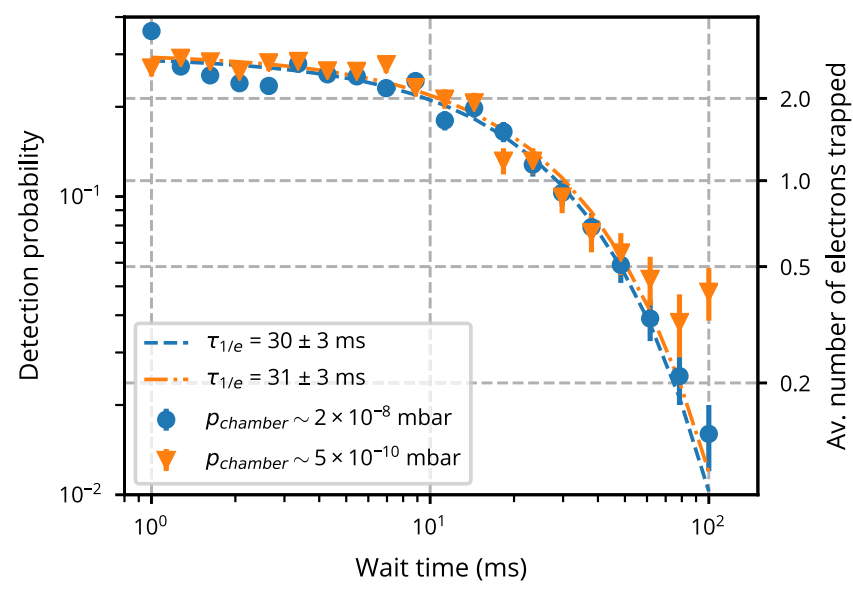

FIG. 8. Electron storage measurements with different background pressure. Data shown as filled circles (fitted by the dashed curve) are taken at typical operating conditions, where the ion pump is switched off. Data shown as triangles (fitted by the dashdotted curve) are taken at lower chamber pressure, which is achieved by running the ion pump.

magnitude, lowering the signal-to-noise ratio of our measurements. Hence, we typically operate with the ion pump switched off during measurements. The pressure in the chamber then increases above $1 \times 10^{-8}$ mbar.

Figure 8 shows two electron storage time measurements. The data shown as circles are taken under typical conditions, where the ion pump is switched off and the chamber pressure is about $2 \times 10^{-8}$ mbar. Turning on the ion pump and repeating the measurement, we record the data shown as triangles. To account for the detections due to the ion pump, the background level is measured separately and subtracted from this data set. As the storage times agree within the measurement uncertainties, we rule out collisions with background gas as the dominant loss mechanism.

\section{Stability of electron motion}

Here, we evaluate the stability of electron trajectories in the trap by numerical integration of the electron motion. As discussed earlier, the energy, and consequentially the trajectory, of a single electron is determined by the surplus ionization energy, the ionization location, and the phase of the microwave field at ionization. Our primary aim will be to illustrate the electron loss mechanisms rather than attempting to fully reproduce our experiments in simulation, so we simplify the computational problem by considering motion along just one axis and ignoring the energy due to the ionization process. The latter is likely small compared to the energy of the pseudopotential and will not advance our understanding much. The path of the ionization lasers follows the $x$ axis when projected on the $x y$ plane of the pseudopotential [cf. Fig. 2(a)], and, based on neutral calcium fluorescence measurements, we know electrons are created up to several millimeters away from the trap center. Hence, we choose to simulate the motion of a single charge in the time-dependent field from the microwave electrode along the $x$ axis. The simulation variables are the ionization distance from the trap center and the phase of the microwave drive at the ionization time.

Figure 9 displays the simulation results. The three panels share $x$ and $y$ axes. In Fig. 9(a), we show a map of the storage time in the trap. The calculated storage time is capped at $1 \mathrm{~ms}$ to keep calculation times reasonable. Trajectories that live up to this time are likely stable in our simulation indefinitely. We see that electron trajectories are universally stable for ionization distances up to about $120 \mu \mathrm{m}$ from the trap center. Beyond that distance, the storage time depends strongly on the microwave phase, ranging from subnanoseconds to more than $1 \mathrm{~ms}$. The transition from stable to unstable trajectories is very pronounced for all microwave phases.

The reason behind this striking behavior becomes clearer when we look at the frequency of the secular motion and its amplitude, which are presented in Figs. 9(b) and 9(c). Frequencies and amplitudes are only shown where the storage time in the trap exceeds $1 \mu \mathrm{s}$; white areas indicate more rapid electron loss. The transition to unstable trajectories in Fig. 9(b) happens at the same secular frequency for all parameter combinations (see dashed contour line), and we can identify this frequency as the seventh subharmonic of the microwave drive frequency: $1.6 \mathrm{GHz} / 7 \approx 229 \mathrm{MHz}$. The sixth subharmonic around $267 \mathrm{MHz}$ is also visible as a unicolored band in parameter space, where the secular frequency is locked to the subharmonic (see also Fig. 10). At these subharmonic frequencies, energy is pumped from the driven micromotion into the secular motion, which heats up the motion. We can observe the heating effect on the map of motion amplitudes in Fig. 9(c): The motion amplitude first increases smoothly as a function of the ionization distance from the trap center, until an amplitude of about $250 \mu \mathrm{m}$ is reached (see labeled contour). Here, the motion amplitude suddenly increases. This jump coincides with the secular frequency hitting the sixth subharmonic. The trap anharmonicity is not sufficient to drive electrons out of the trap, however. Electron loss occurs for motion amplitudes just above $400 \mu \mathrm{m}$, when the seventh subharmonic is reached.

The interplay of motion amplitude and frequency is more easily visualized when we pick a single phase of the microwave drive. Figure 10 displays the motion amplitude (blue dots, left axis) and the secular frequency (orange diamonds, right axis) for a microwave phase at an ionization of $\phi=0.35 \times \pi$. The sixth and seventh subharmonic frequencies are indicated by the dashed lines. As described before, the frequency locking to the sixth subharmonic is accompanied by a sudden increase in the motion amplitude, 

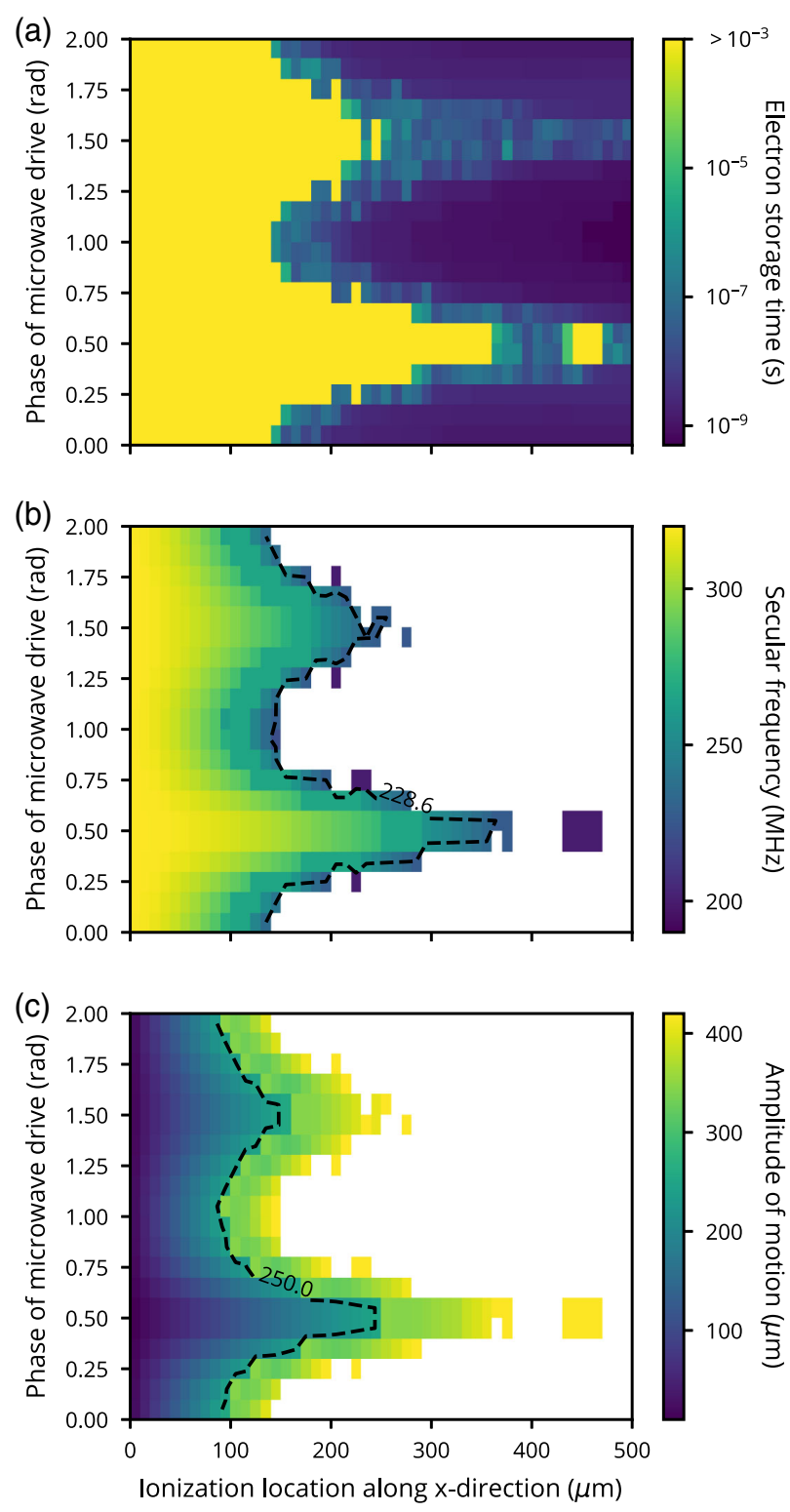

FIG. 9. Electron motion simulations. (a) Storage times for onedimensional motion through the trap along the $x$ axis and the trap center [see Figs. 2(a) and 2(b)]. Storage times are calculated for a maximum time of $1 \mathrm{~ms}$. The plot axes indicate the ionization location and the microwave phase at the time of ionization. The electron is assumed to have zero kinetic energy at the time of ionization. (b) Secular frequency of motion. The dashed curve highlights the frequency contour for the seventh subharmonic of the microwave drive frequency. (c) Corresponding motion amplitudes. The dashed contour line for an amplitude of $250 \mu \mathrm{m}$ highlights where the motion frequency coincides with the sixth subharmonic of the drive frequency and the motion amplitude increases abruptly. White areas in panels (b) and (c) indicates storage times below $1 \mu \mathrm{s}$.

and electron loss occurs when the secular frequency coincides with the seventh subharmonic of the drive frequency.

The analysis of one-dimensional electron motion illustrates the relevance of nonlinear resonances for anharmonic

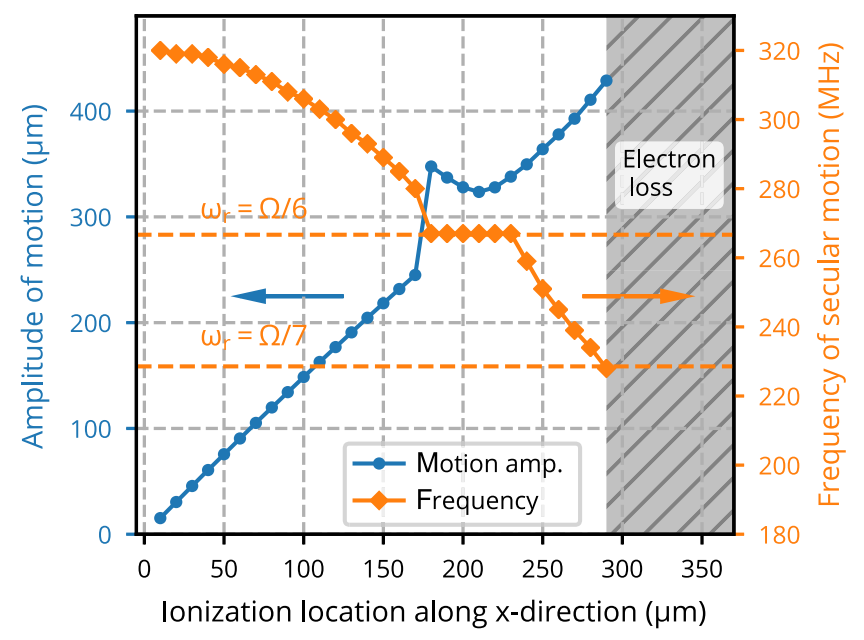

FIG. 10. Amplitude (left axis, blue dots) and frequency (right axis, orange diamonds) of electron motion as a function of ionization location for a microwave drive phase of $\phi=0.35 \times \pi$ at ionization. The orange dashed lines indicate subharmonic resonance frequencies. Electron motion becomes unstable when the secular frequency reaches the seventh subharmonic, corresponding to a motion amplitude of about $420 \mu \mathrm{m}$. For ionization distances from the trap center of about 180-230 $\mu$ m, we confirm that the subharmonically driven motion is accompanied by an increase in the motion amplitude.

potentials. On a qualitative level, it is straightforward to extrapolate from one to three dimensions. Since all modes of motion are coupled, the density of nonlinear resonances in frequency space inflates [39]. Energy can be pumped from the driving field into the electron secular motion at a wide range of frequencies. While many electron trajectories are stable in simulations, like for the sixth subharmonic discussed earlier, the trajectories are not robust to perturbations when a cooling mechanism is not present at the same time. Perturbations to the motion-for instance, due to space charge effects of nearby untrapped or trapped electrons, or charges on the trap itself - or fluctuations in the power of the microwave drive can eventually push an electron into a nonlinear resonance that heats it out of the trap. We believe this process to be at the origin of electron storage times in the millisecond range in our measurements.

Finally, Fig. 9 (and Fig. 10) can also be used to link the width of the resonances observed in the trap frequency measurements to the possible range of electron motion amplitudes. The full width of the radial mode in Fig. 6(b) is about $30 \mathrm{MHz}$, which is roughly the range of frequencies for an electron ionized about $100 \mu \mathrm{m}$ from the trap center, corresponding to a motion amplitude of about $250 \mu \mathrm{m}$.

[1] T. F. Watson, S. G. Philips, E. Kawakami, D. R. Ward, P. Scarlino, M. Veldhorst, D. E. Savage, M. G. Lagally, M. Friesen, S. N. Coppersmith, M. A. Eriksson, and 
L. M. Vandersypen, A Programmable Two-Qubit Quantum Processor in Silicon, Nature (London) 555, 633 (2018).

[2] J. Yoneda, K. Takeda, T. Otsuka, T. Nakajima, M. R. Delbecq, G. Allison, T. Honda, T. Kodera, S. Oda, Y. Hoshi, N. Usami, K. M. Itoh, and S. Tarucha, A QuantumDot Spin Qubit with Coherence Limited by Charge Noise and Fidelity Higher Than 99.9\%, Nat. Nanotechnol. 13, 102 (2018).

[3] A. Laucht, R. Kalra, S. Simmons, J. P. Dehollain, J. T. Muhonen, F. A. Mohiyaddin, S. Freer, F. E. Hudson, K. M. Itoh, D. N. Jamieson, J. C. McCallum, A. S. Dzurak, and A. Morello, A Dressed Spin Qubit in Silicon, Nat. Nanotechnol. 12, 61 (2017).

[4] K. R. Brown, J. Kim, and C. Monroe, Co-designing a Scalable Quantum Computer with Trapped Atomic Ions, npj Quantum Inf. 2, 16034 (2016).

[5] C. D. Bruzewicz, J. Chiaverini, R. McConnell, and J. M. Sage, Trapped-Ion Quantum Computing: Progress and Challenges, Appl. Phys. Rev. 6, 021314 (2019).

[6] A. S. Sørensen and K. Mølmer, Quantum Computation with Ions in Thermal Motion, Phys. Rev. Lett. 82, 1971 (1999).

[7] D. Leibfried, B. DeMarco, V. Meyer, D. Lucas, M. Barrett, J. Britton, W. M. Itano, B. Jelenković, C. Langer, T. Rosenband, and D. J. Wineland, Experimental Demonstration of a Robust, High-Fidelity Geometric Two IonQubit Phase Gate, Nature (London) 422, 412 (2003).

[8] N. Daniilidis, D. J. Gorman, L. Tian, and H. Hartmut, Quantum Information Processing with Trapped Electrons and Superconducting Electronics, New J. Phys. 15, 073017 (2013).

[9] P. Peng, C. Matthiesen, and H. Häffner, Spin Readout of Trapped Electron Qubits, Phys. Rev. A 95, 012312 (2017).

[10] S. Kotler, R. W. Simmonds, D. Leibfried, and D. J. Wineland, Hybrid Quantum Systems with Trapped Charged Particles, Phys. Rev. A 95, 022327 (2017).

[11] T. Ruster, C. T. Schmiegelow, H. Kaufmann, C. Warschburger, F. Schmidt-Kaler, and U. G. Poschinger, A Long-Lived Zeeman Trapped-Ion Qubit, Appl. Phys. B 122, 254 (2016).

[12] N. C. Brown and K. R. Brown, Comparing Zeeman Qubits to Hyperfine Qubits in the Context of the Surface Code: ${ }^{174} \mathrm{Yb}^{+}$and ${ }^{171} \mathrm{Yb}^{+}$, Phys. Rev. A 97, 052301 (2018).

[13] D. Kielpinski, C. Monroe, and D. J. Wineland, Architecture for a Large-Scale Ion-Trap Quantum Computer, Nature (London) 417, 709 (2002).

[14] F. Mintert and C. Wunderlich, Ion-Trap Quantum Logic Using Long-Wavelength Radiation, Phys. Rev. Lett. 87, 257904 (2001).

[15] C. Piltz, T. Sriarunothai, S.S. Ivanov, S. Wölk, and C. Wunderlich, Versatile Microwave-Driven Trapped Ion Spin System for Quantum Information Processing, Sci. Adv. 2, e1600093 (2016).

[16] C. Ospelkaus, U. Warring, Y. Colombe, K. R. Brown, J. M. Amini, D. Leibfried, and D. J. Wineland, Microwave Quantum Logic Gates for Trapped Ions, Nature (London) 476, 181 (2011).

[17] T. P. Harty, M. A. Sepiol, D. T. C. Allcock, C. J. Ballance, J. E. Tarlton, and D. M. Lucas, High-Fidelity Trapped-Ion Quantum Logic Using Near-Field Microwaves, Phys. Rev. Lett. 117, 140501 (2016).
[18] K. A. Twedt and S. L. Rolston, Electronic Detection of Collective Modes of an Ultracold Plasma, Phys. Rev. Lett. 108, 065003 (2012).

[19] P. Kruit, R. G. Hobbs, C. S. Kim, Y. Yang, V. R. Manfrinato, J. Hammer, S. Thomas, P. Weber, B. Klopfer, C. Kohstall, T. Juffmann, M. A. Kasevich, P. Hommelhoff, and K. K. Berggren, Designs for a Quantum Electron Microscope, Ultramicroscopy 164, 31 (2016).

[20] S. C. Burd, R. Srinivas, J. J. Bollinger, A. C. Wilson, D. J. Wineland, D. Leibfried, D. H. Slichter, and D. T. Allcock, Quantum Amplification of Mechanical Oscillator Motion, Science 364, 1163 (2019).

[21] D. J. Wineland, P. Ekstrom, and H. Dehmelt, Monoelectron Oscillator, Phys. Rev. Lett. 31, 1279 (1973).

[22] G. Ciaramicoli, I. Marzoli, and P. Tombesi, Scalable Quantum Processor with Trapped Electrons, Phys. Rev. Lett. 91, 017901 (2003).

[23] I. Marzoli, P. Tombesi, G. Ciaramicoli, G. Werth, P. Bushev, S. Stahl, F. Schmidt-Kaler, M. Hellwig, C. Henkel, G. Marx, I. Jex, E. Stachowska, G. Szawiola, and A. Walaszyk, Experimental and Theoretical Challenges for the Trapped Electron Quantum Computer, J. Phys. B 42, 154010 (2009).

[24] J. Goldman and G. Gabrielse, Optimized Planar Penning Traps for Quantum-Information Studies, Phys. Rev. A 81, 052335 (2010).

[25] P. Bushev, S. Stahl, R. Natali, G. Marx, E. Stachowska, G. Werth, M. Hellwig, and F. Schmidt-Kaler, Electrons in a Cryogenic Planar Penning Trap and Experimental Challenges for Quantum Processing, Eur. Phys. J. D 50, 97 (2008).

[26] S. A. Lyon, Spin-Based Quantum Computing Using Electrons on Liquid Helium, Phys. Rev. A 74, 052338 (2006).

[27] D. I. Schuster, A. Fragner, M. I. Dykman, S. A. Lyon, and R. J. Schoelkopf, Proposal for Manipulating and Detecting Spin and Orbital States of Trapped Electrons on Helium Using Cavity Quantum Electrodynamics, Phys. Rev. Lett. 105, 040503 (2010).

[28] G. Koolstra, G. Yang, and D. I. Schuster, Coupling a Single Electron on Superfluid Helium to a Superconducting Resonator, Nat. Commun. 10, 5323 (2019).

[29] J. Hoffrogge, R. Fröhlich, M. A. Kasevich, and P. Hommelhoff, Microwave Guiding of Electrons on a Chip, Phys. Rev. Lett. 106, 193001 (2011).

[30] J. Walz, S. B. Ross, C. Zimmermann, L. Ricci, M. Prevedelli, and T. W. Hansch, Combined Trap with the Potential for Antihydrogen Production, Phys. Rev. Lett. 75, 3257 (1995).

[31] R. Alheit, X. Z. Chu, M. Hoefer, M. Holzki, G. Werth, and R. Blumel, Nonlinear Collective Oscillations of an Ion Cloud in a Paul Trap, Phys. Rev. A 56, 4023 (1997).

[32] D. Leibfried, R. Blatt, C. Monroe, and D. Wineland, Quantum Dynamics of Single Trapped Ions, Rev. Mod. Phys. 75, 281 (2003).

[33] M. Goeppl, A. Fragner, M. Baur, R. Bianchetti, S. Filipp, J. M. Fink, P. J. Leek, G. Puebla, L. Steffen, and A. Wallraff, Coplanar Waveguide Resonators for Circuit Quantum Electrodynamics, J. Appl. Phys. 104, 113904 (2008).

[34] H. Malissa, D. I. Schuster, A. M. Tyryshkin, A. A. Houck, S. A. Lyon, H. Malissa, D. I. Schuster, A. M. Tyryshkin, A. A. Houck, and S. A. Lyon, Superconducting Coplanar Waveguide Resonators for Low Temperature Pulsed 
Electron Spin Resonance Spectroscopy, Rev. Sci. Instrum. 84, 025116 (2013).

[35] D. J. Wineland and H. G. Dehmelt, Principles of the Stored Ion Calorimeter, J. Appl. Phys. 46, 919 (1975).

[36] S. Gulde, D. Rotter, P. Barton, F. Schmidt-Kaler, R. Blatt, and W. Hogervorst, Simple and Efficient Photo-Ionization Loading of Ions for Precision Ion-Trapping Experiments, Appl. Phys. B 73, 861 (2001).

[37] D. Gerlich, Inhomogeneous RF Fields: A Versatile Tool for the Study of Processes with Slow Ions, in Advances in Chemical Physics, Vol. 82 (John Wiley \& Sons, New York, 1992), pp. 1-176.

[38] Y. Wang, J. Franzena, and K. P. Wanczekb, The Non-linear Resonance Ion Trap. Part 2. A General Theoretical Analysis, Int. J. Mass Spectrom. Ion Process. 124, 125 (1993).

[39] R. Alheit, S. Kleineidam, F. Vedel, M. Vedel, and G. Werth, Higher Order Non-linear Resonances in a Paul Trap, Int. J. Mass Spectrom. Ion Process. 154, 155 (1996).

[40] J. D. Prestage, A. Williams, L. Maleki, M. J. Djomehri, and E. Harabetian, Dynamics of Charged Particles in a Paul Radio-Frequency Quadrupole Trap, Phys. Rev. Lett. 66, 2964 (1991).

[41] K. Chen, S. T. Sullivan, W. G. Rellergert, and E. R. Hudson, Measurement of the Coulomb Logarithm in a RadioFrequency Paul Trap, Phys. Rev. Lett. 110, 173003 (2013).

[42] K. Mølmer and A. S. Sørensen, Multiparticle Entanglement of Hot Trapped Ions, Phys. Rev. Lett. 82, 1835 (1999).
[43] M. Harlander, R. Lechner, M. Brownnutt, R. Blatt, and W. Hänsel, Trapped-Ion Antennae for the Transmission of Quantum Information, Nature (London) 471, 200 (2011).

[44] K. R. Brown, C. Ospelkaus, Y. Colombe, A. C. Wilson, D. Leibfried, and D. J. Wineland, Coupled Quantized Mechanical Oscillators, Nature (London) 471, 196 (2011).

[45] D. J. Heinzen and D. J. Wineland, Quantum-Limited Cooling and Detection of Radio-Frequency Oscillations by Laser-Cooled Ions, Phys. Rev. A 42, 2977 (1990).

[46] N. Daniilidis, T. Lee, R. Clark, S. Narayanan, and H. Häffner, Wiring Up Trapped Ions to Study Aspects of Quantum Information, J. Phys. B 42, 154012 (2009).

[47] J. Eisert, M. B. Plenio, S. Bose, and J. Hartley, Towards Quantum Entanglement in Nanoelectromechanical Devices, Phys. Rev. Lett. 93, 190402 (2004).

[48] G. Kurizki, P. Bertet, Y. Kubo, K. Mølmer, D. Petrosyan, P. Rabl, and J. Schmiedmayer, Quantum Technologies with Hybrid Systems, Proc. Natl. Acad. Sci. U.S.A. 112, 3866 (2015).

[49] N. Leefer, K. Krimmel, W. Bertsche, D. Budker, J. Fajans, R. Folman, H. Häffner, and F. Schmidt-Kaler, Investigation of Two-Frequency Paul Traps for Antihydrogen Production, Hyperfine Interact. 238, 12 (2017).

[50] M. Ahmadi et al., Investigation of the Fine Structure of Antihydrogen, Nature (London) 578, 375 (2020).

[51] M. Brownnutt, M. Kumph, P. Rabl, and R. Blatt, Ion-Trap Measurements of Electric-Field Noise Near Surfaces, Rev. Mod. Phys. 87, 1419 (2015). 